\title{
State of the Art in der Verhaltensmedizin: Keynote-Beiträge der DGVM-Jahrestagung 2013
}

\author{
Claus Vögele $^{a} \quad$ Bernd Leplow $^{b} \quad$ Andreas Hillert $^{c}$ \\ a Institute for Health and Behaviour, INSIDE, University of Luxembourg, Walferdange, Luxembourg \\ ${ }^{\mathrm{b}}$ Klinische Psychologie, Martin-Luther-Universität Halle-Wittenberg, Halle/Saale, \\ ${ }^{c}$ Schön Klinik Roseneck, Prien am Chiemsee, Deutschland
}

Aktuell sind psychische Störungen für den Hauptanteil der globalen Krankheitslast verantwortlich. Zudem sterben mehr Menschen als je zuvor an chronischen Erkrankungen des Herz-Kreislauf-Systems, der Lunge oder des Stoffwechsels, die zumindest teilweise vermeidbar, vielfach aber in ihrem Verlauf positiv beeinflussbar wären. Psychische Faktoren leisten einen entscheidenden Beitrag zur Entstehung und Aufrechterhaltung dieser und anderer Krankheiten. Würden die Risikofaktoren für diese Erkrankungen durch Verhaltensänderungen reduziert, würde sich die Lebenserwartung weltweit um fast 10 Jahre erhöhen.

Erkenntnisse über die Wechselwirkung von biologischen, psychischen und sozialen Risikofaktoren, wie sie die Verhaltensmedizin zur Verfügung stellt, sind unabdingbar, um wirkungsvolle Präventions- und Behandlungsstrategien zu entwickeln und diese systematisch zu evaluieren [Vögele, 2009]. In diesem thematischen Rahmen stand der letztjährige Kongress der Deutschen Gesellschaft für Verhaltensmedizin und Verhaltensmodifikation (DGVM), dessen State-of-the-Art-Vorträge im vorliegenden Sonderheft der Verhaltenstherapie publiziert und im Folgenden kurz eingeleitet werden:

In den westlichen Industrienationen, aber auch in den Entwicklungsländern, ist in den letzten Jahrzehnten eine enorme Zunahme der Zahl übergewichtiger und adipöser Menschen zu beobachten. Allein in Deutschland hat neueren Studien zufolge nur noch etwa ein Drittel der erwachsenen Bevölkerung ein gesundheitlich wünschenswertes Körpergewicht. Besonders besorgniserregend ist der Umstand, dass auch immer mehr Kinder und Jugendliche übergewichtig sind [Platte et al., 2014]. Übergewicht bzw. Adipositas ist eine chronische Krankheit, die mit einer erheblichen Einschränkung der Lebensqualität einhergeht und zu schweren gesundheitlichen Schäden führen kann. Wie der Beitrag von
Martina de Zwaan und Astrid Müller (Hannover) zeigt, wird für diese Entwicklung in Wechselwirkung mit einer genetischen Vulnerabilität vor allem die leichte Verfügbarkeit hochkalorischer Nahrungsmittel und die Bewegungsarmut in Überflussgesellschaften verantwortlich gemacht. Mit verhaltenstherapeutischen Methoden wird versucht, die Ernährung umzustellen und körperliche Bewegung zu fördern. Der Behandlungserfolg ist dabei abhängig von der Berücksichtigung individueller Komorbiditäten, Risiken, Erwartungen und Ressourcen. Wichtig ist eine realistische Zielsetzung, da der gewünschte Gewichtsverlust oft das Erreichbare übersteigt. Deshalb sollte der Gewichtsverlust nicht das alleinige Therapieziel sein. Nur bei stark ausgeprägter Adipositas werden chirurgische Maßnahmen (Magenverkleinerung) empfohlen. Auch bei diesen Patienten ist die psychologische Betreuung vor und nach dem Eingriff von entscheidender Bedeutung für die langfristige Stabilisierung des Gewichtsverlusts.

Neuere Forschungsergebnisse weisen darauf hin, dass die Vulnerabilität für Übergewicht und Adipositas auch von weiteren Faktoren wie der bakteriellen Besiedelung des Darms, insbesondere des Colons beeinflusst wird [Parekh et al., 2014]. Wie im Beitrag von Ulrich Cuntz (Prien am Chiemsee) diskutiert, steht dieses sogenannte Mikrobiom in einer wechselseitigen Beziehung mit zentralnervös gesteuerten Prozessen, beispielsweise Stress und Angst [Foster und McVey Neufeld, 2013]. Ein besseres Verständnis der funktionellen gastrointestinalen Störungen, z.B. des Reizdarmsyndroms, erfordert offensichtlich auch die Einbeziehung des Mikrobioms und die damit verbundenen immunologischen Vorgänge.

Stress und seine Auswirkungen auf die kardial-vegetative Aktivierung spielen auch bei chronischen Erschöpfungszuständen eine große Rolle [Van Cauwenbergh et al., 2014]. In

\section{KARGER \\ Fax +497614520714}

Information@Karger.com

www.karger.com
(C) 2014 S. Karger GmbH, Freiburg

1016-6262/14/0242-0091\$39.50/0

Accessible online at:

www.karger.com/ver
Prof. Claus Vögele

Université du Luxembourg

Faculté des Lettres, des Sciences Humaines,

des Arts et des Sciences de l'Education

Route de Diekirch, 7220 Walferdange, Luxembourg

Claus.Voegele@uni.lu 
seinem Beitrag diskutiert Jens Gaab (Basel) die aktuelle Literatur zur Behandlung chronischer Erschöpfungszustände und kommt zu dem überraschenden Schluss, dass die wirksamen Behandlungen sich nicht durch die von ihnen postulierten Mechanismen erklären lassen.

Im Beitrag von Bernd Leplow (Halle) geht es um die Darstellung und Diskussion verhaltensmedizinischer Verfahren in der Behandlung und Rehabilitation von neurologischen Patienten. Dabei wird deutlich, dass inzwischen vielfältige Möglichkeiten zur Unterstützung der Selbstregulationsfähigkeit von neurologisch Kranken zur Verfügung stehen, beispielsweise Neurofeedback oder Biofeedback, durch die die Kommunikation ermöglicht und die Verbesserung verlorener oder durch die Erkrankung eingeschränkter kognitiver und motorischer Fähigkeiten erreicht werden kann. Andere Ansätze zielen auf den Umgang mit einer chronischen oder chronischprogredienten neurologischen Erkrankung.

Im letzten Beitrag beleuchtet Ulrike Ehlert (Zürich) schließlich den aktuellen Forschungsstand zur Bedeutung hormoneller Systeme auf das biopsychosoziale Gesamtgefüge im Kontext von Gesundheit und Krankheit. Für die verhal- tensmedizinische Praxis sind diese psychoendokrinologischen Forschungsergebnisse im Rahmen der Differenzialdiagnostik, der Entwicklung individueller Krankheitsmodelle und der Therapieevaluation von Bedeutung.

In der Verhaltensmedizin arbeiten Psychologen und Mediziner gemeinsam an der Erforschung der Bedingungen von Krankheit und Gesundheit. Die Grundlage dafür sind die Erkenntnisse, die aus den experimentellen Analysen des Verhaltens gewonnen werden. Mit diesem Wissen wird zum einen versucht, die Entstehung krankhafter Entwicklungen körperlicher und psychischer Systeme besser zu verstehen. Zum anderen geht es heute zunehmend auch darum, Krankheitsverläufe über systematisch gestaltete Interventionen positiv zu beeinflussen und diese therapeutischen Maßnahmen empirisch zu überprüfen. Wie die im vorliegenden Sonderheft zusammengestellten Beiträge zeigen, ist die Verhaltensmedizin diesen Zielen einen großen Schritt näher gekommen. Die Umsetzung dieser Erkenntnisse hingegen ist eine Herausforderung für uns alle, das mehrdimensional belastete Gesundheitssystem und die Gesellschaft insgesamt. Diesbezüglich sind weitere entschiedene Schritte dringend geboten.

\section{Literatur}

Foster JA, McVey Neufeld KA: Gut-brain axis: how the microbiome influences anxiety and depression. Trends Neurosci 2013;36:305-312.

Parekh PJ, Arusi E, Vinik AI, Johnson DA: The role and influence of gut microbiota in pathogenesis and management of obesity and metabolic syndrome. Front Endocrinol (Lausanne) 2014;7:47.
Platte P, Vögele C, Meule A: Adipositas im Kindes- und Jugendalter: Risikofaktoren, Prävention und Behandlung. Verhaltenstherapie 2014;DOI: 10.1159/000363397 (im Druck).
Van Cauwenbergh D, Nijs J, Kos D, Van Weijnen L, Struyf F, Meeus M: Malfunctioning of the autonomic nervous system in patients with chronic fatigue syndrome: a systematic literature review. Eur J Clin Invest 2014;44:516-526.

Vögele C: Klinische Psychologie: Körperliche Erkrankungen. Weinheim, Beltz, 2009. 